\title{
Next-generation molecular therapy in lung cancer
}

\author{
Jun Qian ${ }^{1,2}$, Pierre P. Massion ${ }^{1,2}$ \\ ${ }^{1}$ Division of Allergy, Pulmonary and Critical Care Medicine, Vanderbilt University Medical Center, Nashville, TN, USA; ${ }^{2}$ Cancer Early Detection \\ and Prevention Initiative, Vanderbilt Ingram Cancer Center, Nashville, TN, USA \\ Correspondence to: Pierre P. Massion, MD. Division of Allergy, Pulmonary and Critical Care Medicine, Vanderbilt University Medical Center, \\ Nashville, TN 37232, USA; Cancer Early Detection and Prevention Initiative, Vanderbilt Ingram Cancer Center, Nashville, TN 37232 , USA. \\ Email: pierre.massion@vanderbilt.edu. \\ Provenance: This is an invited Editorial commissioned by Guest Section Editor Dr. Chunlin Ou, MD, PhD (Cancer Research Institute of Central \\ South University, Changsha, China). \\ Comment on: Campbell JD, Alexandrov A, Kim J, et al. Distinct patterns of somatic genome alterations in lung adenocarcinomas and squamous cell \\ carcinomas. Nat Genet 2016;48:607-16.
}

Submitted Dec 20, 2017. Accepted for publication Dec 27, 2017.

doi: $10.21037 /$ tlcr.2018.01.03

View this article at: http://dx.doi.org/10.21037/tlcr.2018.01.03

The identification of molecular subsets of non-small cell lung cancer (NSCLC) has completely modified our approach to the management of this disease. NSCLC is the most common type of lung cancer (80-85\%), which is the number one cancer killer in the nation and worldwide (1). NSCLC can be further pathologically divided into two main subtypes including adenocarcinoma (ADC, 40\%) and squamous cell carcinoma (SCC, 25\%). Recent advances in genomic mutational analysis and targeted therapy have made significant progress in the treatment of lung ADC patients, especially for those patients with certain specific activating mutations in EGFR, $A L K$ and ROS1 (2). In contrast, no molecular targeted therapy has been specifically approved for the treatment of patients with SCC of the lung (3). Despite recent substantial advances for the treatment of lung cancer targeting $T$ cell checkpoint molecule CTLA-4 or PD-1/PD-L1 (4), promising new targets and therapy strategies are still critically needed. Genome-sequencing of a large sample of NSCLC specimens may facilitate the identification of novel molecular targets and development of novel therapies in order to improve the outcomes of lung cancer patients.

In the May 2016 issue of Nature Genetics, Campbell et al. reported the results from analyzing exome sequences and copy number profiles of 1,144 lung cancers including $660 \mathrm{ADC}$ and 484 SCC (5). They identified 38 significantly mutated genes in lung ADC and 20 significantly mutated genes in lung SCC. Fourteen genes were found mutated across tumor types but not identified when analyzed separately. With this large data set, low-frequency alterations in critical oncogenic pathways such as RTK/ RAS/RAF pathway genes (SOS1 and RASA1) and Rho kinase signaling genes (VAV1 and ARHGAP35) were identified. Only six genes such as TP53, RB1, ARID1A, $C D K N 2 A$, PIK3CA, and NF1 were significantly mutated in both tumor types, suggesting that somatic alterations can have different oncogenic potential in different cellular contexts. Further, they reported new significantly mutated genes including PPP $3 C A, D O T 1 L$, and FTSFD1 in lung $\mathrm{ADC}$; RASA1 in lung SCC; and KLF5, EP300, and CREBBP in both tumor types. New amplification peaks contain MIR21 in lung ADC, MIR205 in lung SCC, and MAPK1 in both. These findings contribute to the characterization of a very heterogeneous tumorigenic process in two main subtypes of lung cancer, that reflect individual genetic predisposition, different progenitor cells, the stochastic acquisition of mutational events that later is selected for oncogenic drivers from which we derive tremendous insights to better manage these patients and to design future molecular therapies.

\section{Newly identified mutation drivers}

In 2014, The Cancer Genome Atlas Research Network (TCGA) reported $75 \%$ of lung ADC had oncogenic activities that are drivers of the RTK/RAS/RAF activation 
pathway by adding amplifications in MET and ERBB2 as well as mutations of NF1 and RIT1 as driver events specifically in otherwise oncogene-negative lung ADC (6). Campbell et al. have now reported newly identified driver genes such as RASA1, SOS1, VAV1 and ARHGAP35 although the total proportion of oncogene-positive lung ADC remains almost same $(76 \%)$, leaving up to $24 \%$ of lung ADCs still with unknown or undetected alteration in the RTK/Ras/Raf pathway (5). Interestingly, both CREBBP and EP300 are epigenetic modifier and collectively mutated in up to $18 \%$ of small cell lung cancer (SCLC) (7), suggesting epigenetic alterations as common mechanistic driver in lung cancer. It is worth noting that the majority of previously oncogenenegative lung ADC (12\%) harbors NF1 mutations, which is consistent with the TCGA study. NF1 is a negative regulator of the Ras cellular proliferation pathway and also exerts tumor suppression via other mechanisms. NF1 is 1 of 6 genes significantly mutated in both subtypes, implying a potential driver pathway that warrants further study in both subtypes. Nonetheless, the number clinical trials of NF1-based therapeutic approaches are currently limited (8). Improved understanding of the implications of NF1 aberrations is critical for the development of novel therapeutic strategies. In contrast, SOS1 is a major member that transduces receptor tyrosine kinase signaling to Ras and gain-of-function mutations. These mutations were found in SOS1 and proposed to be a new mechanism by which upregulation of the Ras pathway is independent of PTPN11 or KRAS mutation in Noonan syndrome (9). A small molecule inhibitor targeting SOS1 has been reported recently and might be useful as both biological tools and future therapeutic development (10). This study illustrates nicely the benefit of increasing sample size to discover new mutations and molecular alterations potentially targetable. Considering that the genomic landscape is markedly distinct in never-smokers compared to smokers (11), further testing of a dose response association between smoking history and genomic signature (12) could uncover different mechanisms of tumorigenesis and guide therapy. In addition, testing the association between specific genetic signatures in tumor DNA and genetic predisposition measured by genomewide association study (GWAS) in germline DNA may shed light in the mechanisms of lung cancer development among never smokers, a much-understudied population.

\section{New immunotherapy targets?}

Immunotherapies targeting $\mathrm{T}$ cell checkpoint molecule including CTLA-4 and PD-1/L1 have demonstrated therapeutic efficacy in a wide spectrum of human malignancies including NSCLC (13). Yet the majority of patients with NSCLC (70-80\%) do not respond to PD-1/L1 inhibitor therapy. This may be in part because we lack a reliable biomarker predictive of response to PD-1/L1 therapy (4). The success of immunotherapy is thought to benefit from targeting tumor specific antigens. In this context, neoantigens encoded by tumor-specific mutated genes represent attractive targets for $\mathrm{T}$ cellbased cancer immunotherapy (14). In Campbell's study, the authors comprehensively analyzed the potential immunogenic properties of the mutational landscape in both subtypes of NSCLC. Overall, $47 \%$ of lung ADC and $53 \%$ of lung SCC samples had at least five predicted neoantigens, suggesting potential for immunotherapy. The predicted number of neoantigens was greater amongst smokers $v s$. never smokers. This supports the previous observation that NSCLC from smokers may respond better to immune therapy (15). Some highly recurrent mutations that were predicted to result in neoepitopes may have histological specificity with TP53 p.V157f, p.R175G, p.P278A, PIK3CA p.E542K and NFE2L2 p.E79Q, which is more prevalent in lung SCC while in contrast, $B$-Raf p.G466v and EGFR p.G719A is more prevalent in lung ADC. Future studies are needed to link these candidates with clinical outcomes and responses to immune checkpoint inhibitors or to test them in vaccine based therapies. The most recent approval of chimeric antigen receptor (CAR) T-cell therapy on acute lymphoblastic leukemia (ALL) patients further raises the potential of such therapy against solid tumors (16). As we gain a better understanding of the role of patient-specific mutations and neoantigens in NSCLC, it is likely that we will need to validate some combination of targeting molecular drivers and of using neoantigens specific immune therapeutic strategy.

\section{Future directions}

Several key areas of intense research activity are likely to improve our understanding of and include the study of tumor heterogeneity, single cell analysis and functional genomic approaches. Considerable progress has been made in our understanding of tumor heterogeneity and how it will require adequate sampling and probably whole genome sequencing to identify and further understand the regulatory mechanisms driving evolution of cancer drivers 
in clonal and subclonal populations. Strategies to clinically translate methods of assessing clonality, spatial and temporal heterogeneity is under investigation (17-20). The promise of single cell analysis may help us understand how the complexity of the human tumors arises from cells that are spun off through cell division from progenitor cells in the clonal and subclonal populations of the tumor and further explore tumor heterogeneity. Whole exome sequencing of protein encoding genes has brought major determinants of tumor behavior to light but does not deliver functional alterations in lung cancer (21). A large scale proteomic analysis lead by Berger $e t a l$. followed the study of interest and demonstrated the feasibility of systematic functional interpretation of the cancer genome (21). They analyzed non-canonical variants as meaningful oncogenic drivers that were not identified in the past. Using an expression-based variant impact phenotyping (eVIP) method that uses gene expression changes to distinguish impactful from neutral somatic mutations, they selected candidates followed by a gain of function cancer phenotype screen in mice. Application of eVIP to lung ADC alleles confirmed 69\% of mutations analyzed as impactful and $31 \%$ as functionally neutral. This study demonstrated the feasibility and the future of systematic functional interpretation of the cancer genome; and, hence may lead to the identification of novel, rare, non-canonical mutations as gain-of-function likely "driver" oncogenic mutations that could be targeted in the future.

\section{Acknowledgements}

Funding: This work was supported by grants from the National Cancer Institute (NCI) (No. CA 196405, CA 163772).

\section{Footnote}

Conflicts of Interest: The authors have no conflicts of interest to declare.

\section{References}

1. Siegel R, Ma J, Zou Z, et al. Cancer statistics, 2014. CA Cancer J Clin 2014;64:9-29.

2. Chan BA, Hughes BG. Targeted therapy for non-small cell lung cancer: current standards and the promise of the future. Transl Lung Cancer Res 2015;4:36-54.

3. Liao RG, Watanabe H, Meyerson M, et al. Targeted therapy for squamous cell lung cancer. Lung Cancer
Manag 2012;1:293-300.

4. Somasundaram A, Burns TF. The next generation of immunotherapy: keeping lung cancer in check. J Hematol Oncol 2017;10:87.

5. Campbell JD, Alexandrov A, Kim J, et al. Distinct patterns of somatic genome alterations in lung adenocarcinomas and squamous cell carcinomas. Nat Genet 2016;48:607-16.

6. Cancer Genome Atlas Research Network. Comprehensive molecular profiling of lung adenocarcinoma. Nature 2014;511:543-50.

7. George J, Lim JS, Jang SJ, et al. Comprehensive genomic profiles of small cell lung cancer. Nature 2015;524:47-53.

8. Yap YS, McPherson JR, Ong CK, et al. The NF1 gene revisited - from bench to bedside. Oncotarget 2014;5:5873-92.

9. Tartaglia M, Pennacchio LA, Zhao C, et al. Gain-offunction SOS1 mutations cause a distinctive form of Noonan syndrome. Nat Genet 2007;39:75-9.

10. Evelyn CR, Duan X, Biesiada J, et al. Rational design of small molecule inhibitors targeting the Ras GEF, SOS1. Chem Biol 2014;21:1618-28.

11. Govindan R, Ding L, Griffith M, et al. Genomic landscape of non-small cell lung cancer in smokers and neversmokers. Cell 2012;150:1121-34.

12. Alexandrov LB, Ju YS, Haase K, et al. Mutational signatures associated with tobacco smoking in human cancer. Science 2016;354:618-22.

13. Chen L, Han X. Anti-PD-1/PD-L1 therapy of human cancer: past, present, and future. J Clin Invest 2015;125:3384-91.

14. Schumacher TN, Schreiber RD. Neoantigens in cancer immunotherapy. Science 2015;348:69-74.

15. Nogrady B. Immunotherapy: Chemical tricks. Nature 2014;513:S10-1.

16. Yong CS, Dardalhon V, Devaud C, et al. CAR T-cell therapy of solid tumors. Immunol Cell Biol 2017;95:356-63.

17. Jamal-Hanjani M, Wilson GA, McGranahan N, et al. Tracking the Evolution of Non-Small-Cell Lung Cancer. N Engl J Med 2017;376:2109-21.

18. McGranahan N, Favero F, de Bruin EC, et al. Clonal status of actionable driver events and the timing of mutational processes in cancer evolution. Sci Transl Med 2015;7:283ra54.

19. Gerlinger M, Rowan AJ, Horswell S, et al. Intratumor heterogeneity and branched evolution revealed by multiregion sequencing. N Engl J Med 2012;366:883-92 . 
20. Abbosh C, Birkbak NJ, Wilson GA, et al. Phylogenetic ctDNA analysis depicts early-stage lung cancer evolution. Nature 2017;545:446-51.

Cite this article as: Qian J, Massion PP. Next-generation molecular therapy in lung cancer. Transl Lung Cancer Res 2018;7(Suppl 1):S31-S34. doi: 10.21037/tlcr.2018.01.03
21. Berger AH, Brooks AN, Wu X, et al. High-throughput Phenotyping of Lung Cancer Somatic Mutations. Cancer Cell 2016;30:214-28. 